\title{
Acoustic Response to the Action of Nanosecond Laser Pulses on an In/CdTe Thin-Film Heterostructure
}

\author{
A. I. Vlasenko ${ }^{a}$, V. P. Veleshchuk ${ }^{a}$, V. A. Gnatyuk ${ }^{a}$, S. N. Levitskii ${ }^{a}$, \\ Z. K. Vlasenko ${ }^{a}$, G. D. Ivlev ${ }^{b}$, and E. I. Gatskevich ${ }^{c}$ \\ ${ }^{a}$ Lashkaryov Institute of Semiconductor Physics, National Academy of Sciences of Ukraine, \\ pr. Nauki 41, Kyiv, 03028 Ukraine \\ ${ }^{b}$ Belarusian State University, pr. Nezavisimosti 4, Minsk, 220030 Belarus \\ $c$ Belarusian National Technical University, pr. Nezavisimosti 65, Minsk, 220013 Belarus \\ e-mail:vvvit@ukr.net \\ Received June 27, 2014; in final form, December 3, 2014
}

\begin{abstract}
The photothermoacoustic method has been used for diagnostics of thermobarodynamic processes in the metal $\operatorname{In}(400 \mathrm{~nm}) /$ semiconductor $(\mathrm{CdTe})$ thin-film system under nanosecond laser irradiation $(7 \mathrm{~ns}$, $\lambda=532 \mathrm{~nm}$ ) in natural conditions (in air) and in a liquid medium (water). From the analysis of the data obtained, the dependence of the pressure induced in the energy-release region on the irradiation energy density has been established and the melting threshold of In film has been determined. Under irradiation of In/CdTe in water, the pressure is higher than in air: 17 times higher at the melting threshold of In film and 30 times higher at twice the temperature. It has been found that the laser pulse treatment of In/CdTe/Au samples in water makes it possible to obtain diode structures with better parameters: smaller leak currents and a steeper current-voltage characteristic under the forward bias of the $p-n$ junction.
\end{abstract}

DOI: $10.1134 / \mathrm{S} 1063783415060347$

\section{INTRODUCTION}

One of the promising methods for the formation of shallow and abrupt $p-n$ junctions, e.g., for the development of CdTe diodes employed in detectors of ionizing radiation, is the nanosecond laser irradiation of an In/CdTe film structure $[1,2]$. Instead of the thermal mechanism, this method implements the barodiffusion mechanism of introducing indium impurities into CdTe due to high pressure gradients [3, 4].

In order to increase the diffusion coefficient and the concentration of electrically active dopant atoms, one has to increase the energy density $E$ in a laser pulse. However, this leads to formation of undesirable defects in the zone of the laser doping of the semiconductor, which is one of the disadvantages of this method being implemented in ordinary conditions of irradiation of In/CdTe, i.e., in the air atmosphere. If the structure is irradiated in fluids transparent for a laser beam, e.g., in water, sharp gradients of pressure are possible at smaller $E$, without significant heating of the CdTe crystal surface and undesirable defect formation.

In experiments in this line of research, it is important to control the value of the pressure $P$ arising as a result of laser-stimulated nanosecond processes of heating, melting, and gas and plasma formation proceeding near the original (abrupt) $\mathrm{H}_{2} \mathrm{O}$ /In interface. The initiation of these processes with an increase in $E$, in particular, when the melting point of In film is reached, can lead to a significant stepwise increase in $P$. In this case, the melting point of indium under irradiation of In/CdTe in conditions of a "clamped" surface can shift toward greater values due to the dependence on pressure. This raises the problem of determining the melting threshold of In, which is solved in this work via the application of the photothermoacoustic (PTA) method [5-9] for diagnostics of the laser action.

The aim of the present work is to study by this method in situ the thermobarodynamic processes initiated by the action of nanosecond laser pulses under irradiation of an In/CdTe film heterostructure in natural conditions (in air) and in fluid (water).

\section{SAMPLE PREPARATION AND EXPERIMENTAL TECHNIQUE}

As a substrate material for obtaining In/CdTe heterostructures, we used $p$-CdTe(111) plates with the dimensions of $5 \mathrm{~mm} \times 5 \mathrm{~mm}$ (with a thickness of $0.3-$ $0.5 \mathrm{~mm}$ ) and a resistivity of $(2-4) \times 10^{9} \Omega \mathrm{cm}$. Before applying indium film, the surface of the plates was polished mechanically and then treated in a polishing etch. The 400-nm-thick In film, which later served as an electric contact for measuring the current-voltage characteristics of the structures, and the second $\mathrm{Au}$ electrode were applied by vacuum thermal evaporation at a pressure of $10^{-5} \mathrm{~atm}$. 


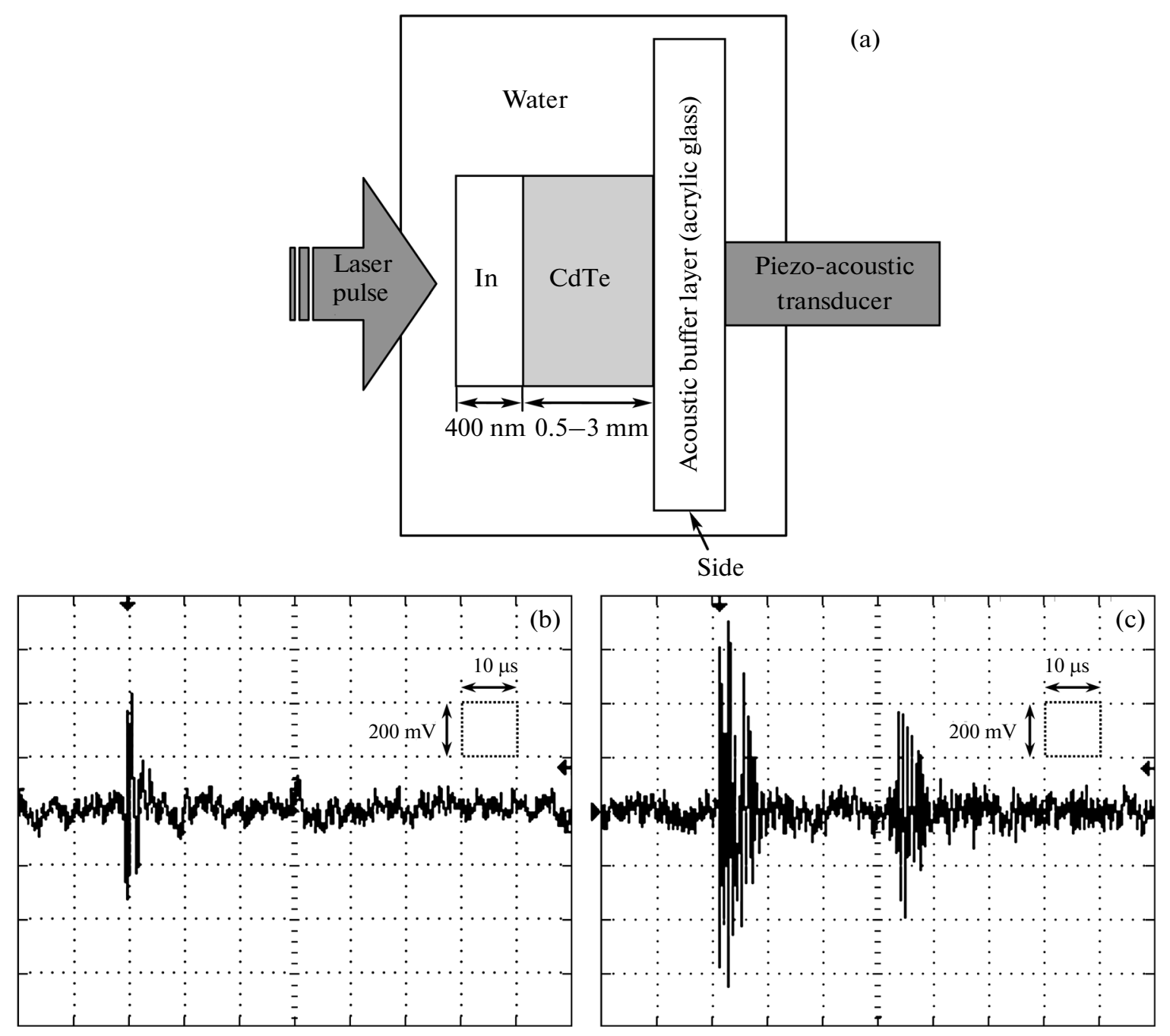

Fig. 1. (a) Scheme of the experiment on irradiation in water using the PTA method and (b, c) signals from the piezoelectric detector under laser irradiation of In/CdTe samples in water at $E_{\mathrm{inc}}=$ (b) 1.2 and (c) $20.5 \mathrm{~mJ} / \mathrm{cm}^{2}$.

We studied the influence of single pulses of secondharmonic radiation from a YAG : $\mathrm{Nd}$ laser $(\lambda=$ $532 \mathrm{~nm}, \tau_{\text {imp }}=7 \mathrm{~ns}$ ) on In/CdTe samples in air and in a cuvette with distilled water. The diameter of the laser spot (irradiated zone) was $1 \mathrm{~mm}$, and the energy distribution over its area was sufficiently uniform. The value of $E$ was varied by calibration filters.

The acoustic response to the laser action was detected by a piezoelectric transducer on the back side of the sample. The piezoelectric detector (of type P113 from the package of the AF-15 acoustic device) was a TsTS-18 piezoelectric element in the form of a truncated cone in a metal casing with a ceramic protector for the acoustic contact. The resonance frequency was $400 \mathrm{kHz}$. Electric signals from the piezoelectric detector were detected in the frequency range of $200-2000 \mathrm{kHz}$. The instrumental gain factor $\left(A_{\text {in }} / A_{\text {out }}\right)$ of signals was within the linear dynamic range of the amplification circuit and amounted to
50-74 dB. A sample on a perspex substrate (thickness of $1 \mathrm{~cm}$, diameter of $10 \mathrm{~cm}$ ) and a piezoelectric transducer were placed into a copper cell with water (Fig. 1a). The measured absorption coefficient of the input glass and water at the wavelength of $532 \mathrm{~nm}$ was $11 \%$.

We also detected flashes of laser plasma by a photo camera with OS 14 and KS 11-19 filters for cutting out the laser radiation. Such plasma, which arises due to the dielectric breakdown in products of evaporation of indium, is quenched in fluid. The morphology of the crystal surface before and after irradiation was controlled by a MI-9 optical microscope.

\section{RESULTS}

The typical acoustic signals under irradiation of an In/CdTe structure in water for two values of the laser pulse energy density $E_{\text {inc }}$ are shown in Figs. $1 \mathrm{~b}$ and 1c. Here, the second pulse corresponds to the reflection from the side face of the acoustic buffer. 
The dependences $A\left(E_{\text {inc }}\right)$ of the amplitude of the acoustic response on the laser pulse energy density under irradiation of an $\operatorname{In}(400 \mathrm{~nm}) / \mathrm{CdTe}$ structure in water and in air are different (Fig. 2). The curves have a flex point, after which the signal amplitude begins a faster increase with increasing irradiation energy density. Under irradiation of the structure in water, this increase begins from the value of $80 \mathrm{~mJ} / \mathrm{cm}^{2}$, and, under irradiation in air, from $115 \mathrm{~mJ} / \mathrm{cm}^{2}$.

The irradiation energy density at which the inclination of the curve changes corresponds to the melting threshold of indium film, because, at the instant of melting, a sharp increase in the volume takes place [7, 8]. Under irradiation in water, the melting threshold is somewhat lower.

In the process of laser radiation, the parameters of indium and the neighboring water layer (the radiation absorption coefficients, thermal conductivity coefficients, etc.) can change, which leads to thermal nonlinearity [7, 10-13]. However, under stepwise shock compression at a pressure as high as $27 \mathrm{GPa}$ and temperature as high as $1000 \mathrm{~K}$, the thermal conductivity coefficient of indium is independent of temperature and its three-fold increase is caused only by the variation in the volume upon compression [14].

As follows from Fig. 2, in the region of photothermoacoustic signal, the amplitude $A_{\text {water }}$ exceeds $A_{\text {air }}$ by a factor of 17. Upon irradiation with the energy densities above the melting threshold, e.g., at $E_{\text {inc }}=$ $150 \mathrm{~mJ} / \mathrm{cm}^{2}$, we have $A_{\text {water }} / A_{\text {air }} \approx 30$. The amplitude $A$ is proportional to the transverse deformation component and, correspondingly, the compression.

\section{CALCULATION \\ OF THE PHOTOTHERMOACOUSTIC \\ PRESSURE UNDER NANOSECOND IRRADIATION OF In/CdTe IN WATER}

In the case of a PTA signal when one face is fixed, the pressure was calculated according to the expression $[11,12]$ :

$$
P_{\mathrm{PTA}}(x=0)=\frac{(1-R) I_{\mathrm{inc} \beta_{T}}}{(N+1) C_{p}} .
$$

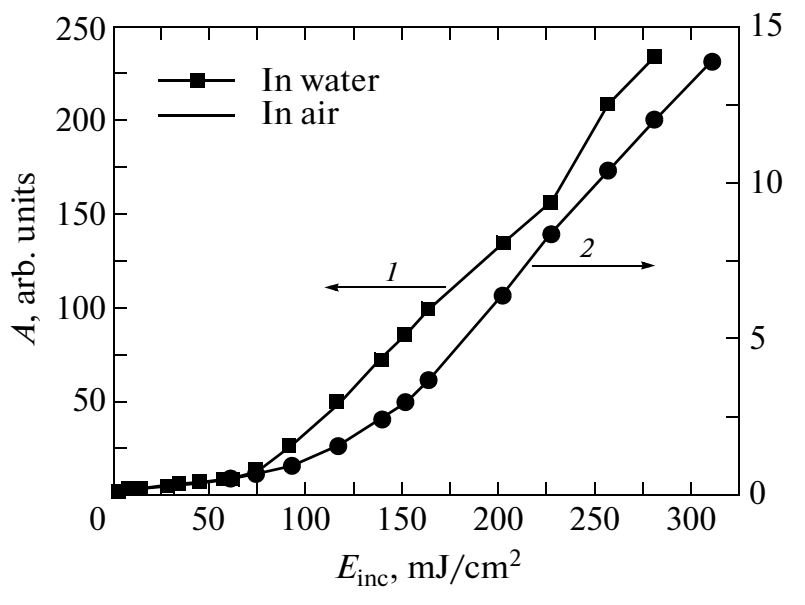

Fig. 2. Dependences of the amplitude of the acoustic response on the irradiation energy of the In/CdTe heterostructure: (1) in water and (2) in air.

Here, we take into account the ratio of the acoustic impedances on the indium-water interface, $N=$ $\rho_{\text {In }} c_{\text {In }} / \rho_{\text {water }} c_{\text {water }}=7.6, I_{\text {inc }}$ is the laser radiation intensity, $\rho$ is the mass density of indium, $c$ is the speed of acoustic wave propagation, $\beta_{T}$ is the coefficient of volume expansion, $\beta=3 \alpha_{T}, C_{p}$ is the heat capacity, and $R$ is the optical reflection coefficient. The physical parameters of In are presented in the table.

Upon melting of In, the pressure $\Delta P_{\text {melt }}$ is affected by the variation in density, $\Delta \rho_{\text {In }}=\rho_{\text {solid }}-\rho_{\text {melt }}$, and the melt front propagation velocity. In the linear approximation with respect to $\Delta \rho_{\text {In }}$, the value of $\Delta P_{\text {melt }}$ is determined by the expression $[15,16]$

$$
\Delta P_{\text {melt }}=\Delta \rho_{\text {In }} d(V h) / d t,
$$

where $V$ is the rate of change in the melt layer thickness $h$. From the numerical solution of the Stefan problem, the dependence of the melt depth $h$ on the time $t$ was calculated. For In/CdTe structures with the film thickness of $400 \mathrm{~nm}$, the velocity of the motion of the interphase boundary to the depth of the sample, $V=$ $d h / d t$, varies from $5.7 \mathrm{~m} / \mathrm{s}$ to $88 \mathrm{~m} / \mathrm{s}$ as $E_{\text {inc }}$ increases from 80 to $1000 \mathrm{~mJ} / \mathrm{cm}^{2}$. The dependence $V(E)$ is close to logarithmic (Fig. 3).

\begin{tabular}{|c|c|c|c|c|c|}
\hline $\begin{array}{l}\text { Sound velocity } \\
\text { (longitudinal } \\
\left.\text { mode } c_{l}\right), \mathrm{m} \mathrm{s}^{-1}\end{array}$ & $\begin{array}{l}\text { Density } \rho \text {, } \\
\mathrm{kg} \mathrm{m}^{-3}\end{array}$ & $\begin{array}{c}\text { Absorption } \\
\text { coefficient } \\
\alpha_{\lambda=0.532, \mathrm{~m}^{-1}}\end{array}$ & $\begin{array}{l}\text { Reflection } \\
\text { coefficient } \\
\qquad R\end{array}$ & $\begin{array}{l}\text { Linear expansion } \\
\text { coefficient } \alpha_{T}, \mathrm{~K}^{-1} \\
\quad(293-333 \mathrm{~K})\end{array}$ & $T_{\text {melt }},{ }^{\circ} \mathrm{C}$ \\
\hline 1400 & 7310 & $6 \times 10^{7}$ & 0.8 & $3.3 \times 10^{-5}$ & 157 \\
\hline $\begin{array}{l}\text { Heat capacity } \\
C_{p}, \mathrm{~J} \mathrm{~kg}^{-1} \mathrm{~K}^{-1}\end{array}$ & $\begin{array}{l}\text { Thermal diffu- } \\
\text { sivity } \chi, \mathrm{m}^{2} \mathrm{~s}^{-1}\end{array}$ & $\begin{array}{l}\text { Thermal conductiv- } \\
\text { ity } \lambda, \mathrm{J} \mathrm{m}^{-1} \mathrm{~s}^{-1} \mathrm{~K}^{-1}\end{array}$ & $\begin{array}{l}\text { Bulk modulus } \\
\qquad E, \mathrm{~Pa}\end{array}$ & $\begin{array}{l}\text { Shear modulus } \\
\qquad G, \mathrm{~Pa}\end{array}$ & $\begin{array}{c}\text { Poisson's ratio } \\
v\end{array}$ \\
\hline 234.46 & $5.0761 \times 10^{-5}$ & 87 & $1.1 \times 10^{10}$ & $4 \times 10^{9}$ & 0.46 \\
\hline
\end{tabular}

Physical parameters of indium 


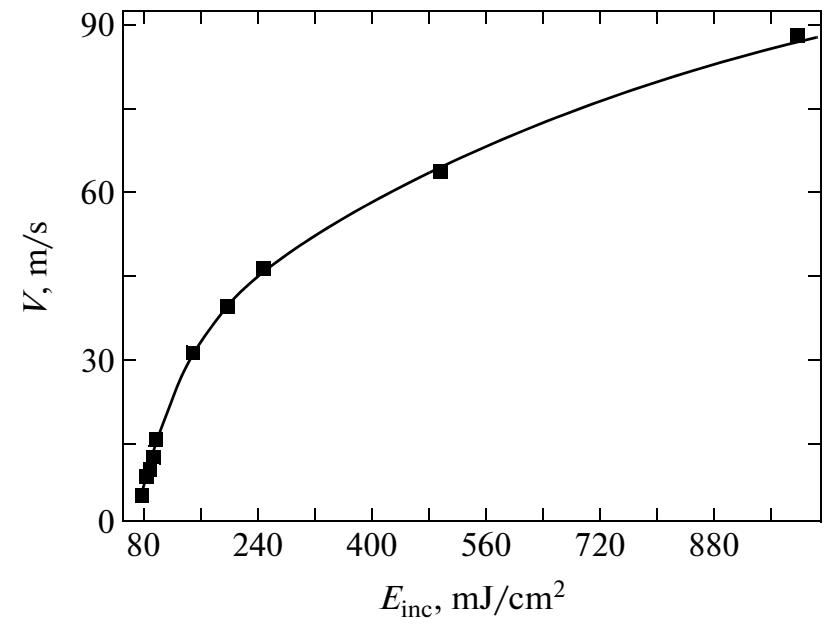

Fig. 3. Maximum velocity of the melt-solid phase front motion for indium film $\left(h_{\mathrm{In}}=400 \mathrm{~nm}\right)$ as a function of the pulse energy $\left(\tau_{\mathrm{imp}}=7 \mathrm{~ns}\right)$ under laser irradiation of In/CdTe by a neodymium laser.

The time dependence of the melt dept, $h(t)$, was also calculated in our work [3]. The quantity $\Delta P_{\text {melt }}$ quadratically depends on $V$, as in $[15,16]$, and increases with an increase in the difference $\Delta \rho_{\text {In }}$. The density of indium upon melting and further heating of the melt $\left(\Delta \rho_{\text {In }}\right.$ in formula (2)) decreases [17], i.e., upon local melting under irradiation, a fast (for a time comparable with $\tau_{\text {imp }}$ ) increase in volume takes place. In this case, $\rho_{\text {In, } T=157^{\circ} \mathrm{C}}=7000 \mathrm{~kg} / \mathrm{m}^{3}, \rho_{\text {In }, T=400^{\circ} \mathrm{C}}=$ $6800 \mathrm{~kg} / \mathrm{m}^{3}$.

From expressions (1) and (2) and taking into account the dependences in Fig. 3, the dependence of the total pressure $P=P_{\mathrm{PTA}}+\Delta P_{\text {melt }}$ on the laser energy density $E_{\text {inc }}$ was calculated (Fig. 4 ). It is evident that the increment of pressure due to melting (expression (2)) is comparable with the PTA signal (expression (1)); in this case, the dependence after the melting threshold is not linear at all (here, the melting threshold $80 \mathrm{~mJ} / \mathrm{cm}^{2}$ was taken from Fig. 2). This dependence is similar to the dependence in Fig. 2. In the calculation, we disregarded the influence of evaporation and instant boiling of water. Taking into account these phenomena, apparently, will lead to an even steeper dependence of pressure on the irradiation energy density [10].

It should also be noted that expression (1) is valid in the case of small pulse energy densities, when the variation in the physical parameters of indium due to the laser action upon the formation of the acoustic response, i.e., before the melting threshold, may be neglected. The approximation of expression (1) for the irradiation energy density above the melting threshold, i.e., without additional mechanisms of acoustic pulse excitation, is shown in Fig. 4, curve 2.

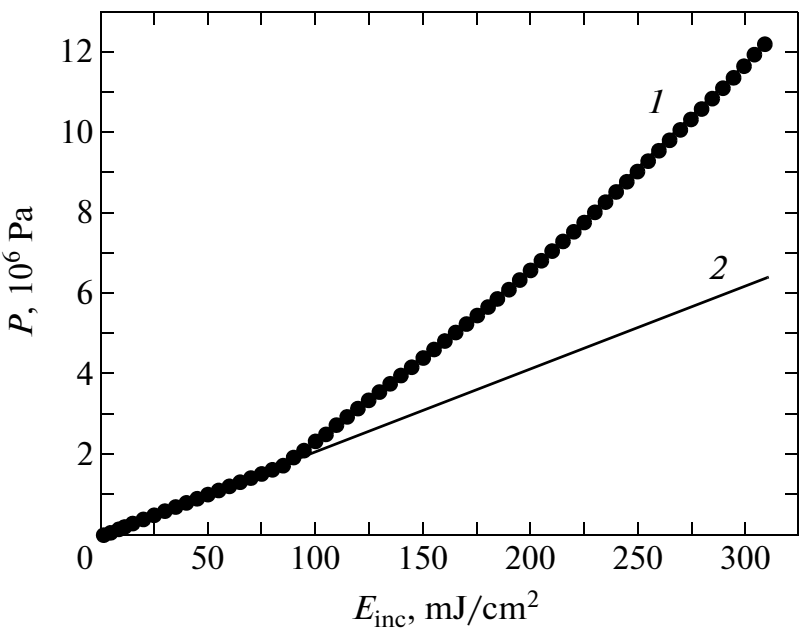

Fig. 4. (1) Pressure $P$ as a function of the incident energy density under laser irradiation of In/CdTe by a neodymium laser ( $\left.\tau_{\mathrm{imp}}=7 \mathrm{~ns}\right)$ in water and (2) approximation of expression (1) above the melting threshold.

Comparing the calculation and experiment, we can obtain the values of pressure: calculating the pressure in the region of small values of PTA signal, e.g., by expression (1) (the linear region up to $E_{\text {inc }}=$ $60 \mathrm{~mJ} / \mathrm{cm}^{2}$, Fig. 4), we can find the pressures for all values of energy in Fig. 2.

In the case of the "shock" deformation, the rate of deformation upon nanosecond irradiation in the case of the PTA effect is described by the expression $\dot{\varepsilon}_{z z}=$ $\frac{1+v}{1-v} \frac{\alpha_{T} \Delta T}{\tau_{\text {imp }}}$ [4]; in this case, the amplitude of the detected photothermoacoustic response signal is proportional to the temperature and deformation increments and inversely proportional to the laser pulse duration [9].

At the values $\tau_{\text {imp }}=7 \mathrm{~ns}$ and $\Delta T=500-1000^{\circ} \mathrm{C}$ for In $(v=0.46)$, we have $\dot{\varepsilon}_{z z} \approx(6-12) \times 10^{6} \mathrm{~s}^{-1}$. The rate of deformation upon melting due to the variation in volume, $\dot{\varepsilon}_{\text {melt }}=\frac{\Delta h / h_{0}}{\tau_{\text {imp }}}$, is also on the order of $10^{6} \mathrm{~s}^{-1}$ (as in $[3,4]$ ). Here, $\Delta h$ is the increment in the linear size and $h_{0}$ is the initial volume of the surface layer, which is easily melted. Thus, at the laser pulse energies employed, the rate of deformation upon melting, $\dot{\varepsilon}_{\text {melt }}$, in the linear approximation is comparable with the rate of deformation under the PTA transformation, $\dot{\varepsilon}_{z z}$, which determines the form of the dependence in Fig. 4. It is an additional argument in favor of that the inflection (variation in the inclination) in Fig. 2 takes place at the irradiation energy density corresponding to the melting threshold of indium. 


\section{CURRENT-VOLTAGE CHARACTERISTICS OF In/CdTe STRUCTURES}

The current-voltage characteristics of In/CdTe samples were measures before and after a single laser irradiation in water and in air. The typical currentvoltage characteristics are demonstrated on the example of structures with 400-nm-thick films (Fig. 5). We see a substantial enhancement of the rectifying properties of the In/CdTe contact after the irradiation, especially in water. The mechanism of doping of the surface layer of CdTe crystals with indium and the formation of the $p-n$ junction are described in [3]. Here, we should only note that the leak current in the reverse current-voltage characteristic under irradiation in water has decreased even more than under irradiation in air and the rectifying voltage has become lower than under irradiation in air (17 V).

\section{DISCUSSION}

Under irradiation of indium film in water, i.e., in the conditions of a clamped face, the dynamics of laser-induced processes in the pulse energy absorption band substantially differs from the dynamics under irradiation in air. Firstly, since water is practically incompressible, the irradiation raises significant pressures, which affect the properties of indium and initiate nonlinear effects. Secondly, under irradiation in water, the evaporation from the indium surface layer is suppressed. Thirdly, a fraction of pulse's energy goes to the heating of the neighboring water layer.

Under irradiation in water, we should take into account the process of water vapor formation. Upon nanosecond laser irradiation of metals placed into water, the so-called explosive boiling of water takes place [10]. This process substantially differs from the ordinary evaporation of water upon reaching the temperature of $100^{\circ} \mathrm{C}$, because vapor has no time to form during the action of a nanosecond pulse. Due to significant pressures in the zone of irradiation, the water boiling point shifts toward higher temperatures. For example, at $P=0.5 \mathrm{MPa}, T_{\text {boil }}=153^{\circ} \mathrm{C}$; at $P=1 \mathrm{MPa}$, $T_{\text {boil }}=180^{\circ} \mathrm{C}$; and an overheated fluid-vapor layer can form $[10,11]$. It should be noted that, according to Fig. 4, at the phase transition point (melting threshold) of indium, the PTA pressure reaches 1.7 MPa and $T_{\text {boil }}$ of water is $204^{\circ} \mathrm{C}$. This value of water boiling temperature is above the melting point of indium $\left(157^{\circ} \mathrm{C}\right)$, which additionally confirms that the increase in the inclination in the dependence $A\left(E_{\text {inc }}\right)$ in Fig. 2 begins as the melting of In begins.

A similar phenomenon was earlier observed upon nanosecond (6 ns) laser irradiation of mercury [11]. Upon clamping of liquid mercury by a quartz plate, the threshold energy density at which boiling begins is $81 \mathrm{~mJ} / \mathrm{cm}^{2}$, which is twice the analogous value for the irradiation in air. In this case, the maximum surface

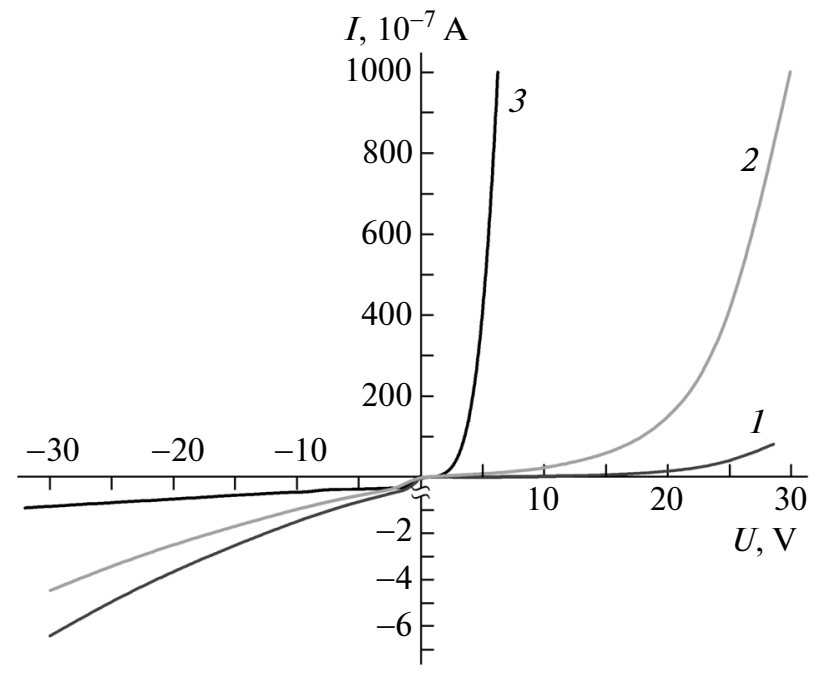

Fig. 5. Current-voltage characteristics of the $\mathrm{In} / \mathrm{CdTe} / \mathrm{Au}$ structure $(1)$ before irradiation and $(2,3)$ after irradiation at $E_{\text {inc }}=100 \mathrm{~mJ} / \mathrm{cm}^{2}(2)$ in air and (3) in water.

temperature exceeds $700^{\circ} \mathrm{C}$, which is twice the boiling point of mercury at normal pressure [11].

Under pulse irradiation in water, due to the dependence on $P$, the melting point of In also increases but, at the values of $P$ of a few megapascals, which are realized in the given case, this increase is small [20]. A substantial (e.g., to $200^{\circ} \mathrm{C}$ ) increase in $T_{\text {melt }}$ of In takes place at huge pressures: $P=10^{9} \mathrm{~Pa}[20]$.

We should take into account the influence of evaporation on the laser-induced processes and the ionization of indium atoms, which are different in air and in water. Under irradiation in air, evaporation proceeds in parallel with melting and laser plasma formation [3]. Under irradiation in water, upon reaching the melting point, there is no evaporation of indium (it is prevented by the water layer); therefore, the laserinduced breakdown is absent. This process begins only at $E_{\text {inc }}=160 \mathrm{~mJ} / \mathrm{cm}^{2}$. The typical luminescence of plasma arising in this case was detected by a photo camera. Below this value of $E_{\text {inc }}$, no flash was observed and, on exceeding $E_{\text {inc }}=160 \mathrm{~mJ} / \mathrm{cm}^{2}$, a bubble at the place of irradiation appeared.

The reduction in the melting point of indium film on a CdTe substrate in water may be explained by the absence of evaporation of indium in water. Due to the laser-induced evaporation of indium atoms in air, significant cooling of the surface takes place; in this case, the heat flux from the In surface can be written as $Q=$ $L_{\text {In }} J_{\text {In }}$, where the mass density flux of evaporated atoms is $J_{\text {In }}(T) \sim P_{\text {pairs }}(T) \sqrt{\frac{M}{2 \pi k_{\mathrm{B}} T}}[18,19] ; M$ is the atomic mass and $L_{\mathrm{In}}$ is the latent heat of evaporation of indium. 
It should be noted that earlier we found by mathematical modeling [3] that an In film with a thickness $h_{\text {In }}=400 \mathrm{~nm}$ under irradiation by a ruby laser (with $\tau_{\text {imp }}=20 \mathrm{~ns}$ ) in air begins melting at $E=16 \mathrm{~mJ} / \mathrm{cm}^{2}$. Taking into account the optical reflection coefficient of indium, $R \approx 0.8$, we obtain $E_{\text {inc }}=80 \mathrm{~mJ} / \mathrm{cm}^{2}$, which is close to the values obtained in this work.

\section{CONCLUSIONS}

(1) We established that, under irradiation of an In/CdTe structure by a neodymium laser $\left(\tau_{\text {imp }}=7 \mathrm{~ns}\right)$ in water, the pressure in the energy-release zone is substantially higher than under irradiation in air. Up to the melting threshold of indium, when the photothermoacoustic effect takes place, the pressures differ by a factor of 17. In the case of the double excess of the melting point of In, the pressures differ by the factor of 30.

(2) It was found that the melting threshold under irradiation of In/CdTe in distilled deionized water by a neodymium laser ( $\tau_{\mathrm{imp}}=7 \mathrm{~ns}$ ) is $E_{\mathrm{inc}}=80 \mathrm{~mJ} / \mathrm{cm}^{2}$, which is smaller than under irradiation in air $\left(115 \mathrm{~mJ} / \mathrm{cm}^{2}\right)$.

(3) The melt front motion velocity $V$ upon nanosecond laser irradiation of an In film $(400 \mathrm{~nm}) / \mathrm{CdTe}$ structure varies from 5.7 to $88 \mathrm{~m} / \mathrm{s}$ as $E_{\text {inc }}$ increases from 80 to $1000 \mathrm{~mJ} / \mathrm{cm}^{2}$ and has a dependence close to logarithmic. At such values of $E_{\text {inc }}$, the additional pressure due to the process of abrupt melting becomes comparable with the photothermoacoustic pressure.

(4) The method of pulsed laser irradiation of In/CdTe in water with the control of pressure from the induced acoustic response made it possible to form diode structures with improved parameters: smaller leak currents and steeper current-voltage characteristics upon the forward bias of the $p-n$ junction.

\section{ACKNOWLEDGMENTS}

This study was supported by the State Fund for Fundamental Research of Ukraine (project no. F54.1/0.33) and the Belarusian Republican Foundation for Fundamental Research (project no. F13K-117).

\section{REFERENCES}

1. V. A. Gnatyuk, T. Aoki, E. V. Grushko, L. A. Kosyachenko, and O. I. Vlasenko, Proc. SPIE-Int. Soc. Opt. Eng. 8142, 81420B-1-7 (2011).
2. V. A. Gnatyuk, T. Aoki, O. I. Vlasenko, and S. N. Levytskyi, in Proceedings of the Nuclear Science Symposium and Medical Imaging Conference Record (NSS/MIC) IEEE 4506, 2011.

3. V. Veleschuk, A. Vlasenko, E. Gatskevich, V. Gnatyuk, G. Ivlev, S. Levytskyi, and T. Aoki, J. Mater. Sci. Eng., B 2 (4), 230 (2012).

4. V. A. Baidulaeva, V. P. Veleshchuk, O. I. Vlasenko, V. A. Gnatyuk, B. K. Dauletmuratov, S. M. Levits'kii, and T. Aoki, Ukr. Fiz. Zh. 56 (2), 171 (2011).

5. V. P. Zharov and V. S. Letokhov, Laser Optical-Acoustic Spectroscopy (Vysshaya Shkola, Moscow, 1984) [in Russian].

6. V. E. Gusev and A. A. Karabutov, Laser Optoacoustics (Nauka, Moscow, 1991) [in Russian].

7. A. A. Karabutov, A. G. Kaptil'nyi, and A. Yu. Ivochkin, High Temp. 45 (5), 613 (2007).

8. A. Baidullaeva, V. P. Veleshchuk, A. I. Vlasenko, B. K. Dauletmuratov, O. V. Lyashenko, and P. E. Mozol', Semiconductors 42 (3), 281 (2008).

9. D. A. Andrusenko, R. M. Burbelo, and A. G. Kuz'mich, Tech. Phys. Lett. 36 (12), 1121 (2010).

10. S. N. Andreev, V. I. Vovchenko, and A. A. Samokhin, Tr. Inst. Obshch. Fiz., Ross. Akad. Nauk 60, 149 (2004).

11. A. A. Karabutov, A. P. Kubyshkin, V. Ya. Panchenko, and N. B. Podymova, Kvantovaya Elektron. (Moscow) 22, 820 (1995).

12. A. A. Karabutov, A. P. Kubyshkin, V. Ya. Panchenko, N. B. Podymova, and E. V. Savateeva, Kvantovaya Elektron. (Moscow) 25, 690 (1998).

13. A. A. Kolomenskii, M. L. Lyamshev, V. G. Mikhalevich, and A. M. Rodin, Izv. Akad. Nauk SSSR, Ser. Fiz. 49, 1121 (1985).

14. A. M. Molodets and A. A. Golyshev, Phys. Solid State 51 (2), 226 (2009).

15. I. A. Veselovskii, B. M. Zhiryakov, N. I. Popov, and A. A. Samokhin, Tr. Inst. Obshch. Fiz., Akad. Nauk SSSR 13, 108 (1988).

16. I. A. Veselovskii, B. M. Zhiryakov, A. I. Korotchenko, and A. A. Samokhin, Kvantovaya Elektron. (Moscow) 12, 381 (1985).

17. B. B. Alchagirov, A. G. Mozgovoi, T. M. Taova, and Kh. B. Khokonov, Perspekt. Mater., No. 3, 33 (2007).

18. S. P. Zhvavyi and G. L. Zykov, Semiconductors 40 (6), 632 (2006).

19. A. A. Kovalev, S. P. Zhvavyi, and G. L. Zykov, Semiconductors 39 (11), 1299 (2005).

20. BSE: Pressure Dependence of the Melting Temperature of Metals. http://bse.sci-lib.com/particle009668. html.

Translated by E. Chernokozhin 